\title{
Demonstration of high-rate laser communications from fast airborne platform: flight campaign and results
}

\author{
Florian Moll*a ${ }^{\mathrm{a}}$, Wolfgang Mitzkus ${ }^{\mathrm{b}}$, Joachim Horwath ${ }^{\mathrm{c}}$, Amita Shrestha ${ }^{\mathrm{a}}$, Martin Brechtelsbauer ${ }^{\mathrm{a}}$, \\ Luis Martin Navajas ${ }^{c}$, Alberto Lozano Souto ${ }^{c}$, Dionisio Diaz Gonzalez ${ }^{c}$ \\ ${ }^{a}$ German Aerospace Center (DLR), Institute of Communications and Navigation, Oberpfaffenhofen, \\ 82234 Wessling, Germany; ${ }^{b}$ Airbus Defence \& Space, Mission Avionics Conceptual Design, \\ Rechliner Straße, 85077 Manching, Germany; ${ }^{\mathrm{c}}$ Vialight Communications GmbH, Friedrichshafener \\ Str.1, 82205 Gilching, Germany
}

\begin{abstract}
Some current and future airborne payloads like high resolution cameras and radar systems need high channel capacity to transmit their data from air to ground in near real-time. Especially in reconnaissance and surveillance missions, it is important to downlink huge amount of data in very short contact times to a ground station during a flyby. Aeronautical laser communications can supply the necessary high data-rates for this purpose. Within the project DODfast (Demonstration of Optical Data link fast) a laser link from a fast flying platform was demonstrated. The flight platform was a Panavia Tornado with the laser communication terminal installed in an attached avionic demonstrator pod. The air interface was a small glass dome protecting the beam steering assembly. All other elements were integrated in a small box inside the Pod's fuselage. The receiver station was DLR's Transportable Optical Ground Station equipped with a free-space receiver front-end. Downlink wavelength for communication and uplink wavelength for beacon laser were chosen from the optical C-band DWDM grid. The test flights were carried out at the end of November 2013 near the Airbus Defence and Space location in Manching, Germany. The campaign successfully demonstrated the maturity and readiness of laser communication with a data-rate of $1.25 \mathrm{Gbit} / \mathrm{s}$ for aircraft downlinks. Pointing, acquisition and tracking performance of the airborne terminal and the ground station could be measured at aircraft speed up to 0.7 Mach and video data from an onboard camera has been transmitted. Link distances with stable tracking were up to $79 \mathrm{~km}$ and distance with data transmission over $50 \mathrm{~km}$. In this paper, we describe the system architecture, the flight campaign and the results.
\end{abstract}

Keywords: Free-space optical communications, aircraft downlink, flight experiment, long range communications, pointing, acquisition and tracking, field demonstration, transportable optical ground station, project DODfast

\section{INTRODUCTION}

State of the art and future airborne high resolution cameras and radar systems have the need for high rate communication links. Particular scenarios, like reconnaissance and surveillance missions, even demand to download huge data chunks from an airborne platform to a ground station during a flyby. Currently, conventional radio-frequency (RF) technology is used for that purpose. However, optical systems can overcome RF systems in terms of lower SWaP (Size, Weight and Power) and the advantage of free and unregulated spectrum use.

In this paper, we report on an experimental demonstration of a high-rate optical downlink from a fast flying platform to a ground station. "Fast" in this context addresses the aircraft speed which is targeted to be 0.7 Mach. The communication channel is chosen to comply with DWDM (Dense Wavelength Division Multiplexing) technology, i.e. the communication wavelength is selected from the DWDM grid in C-band. The experiment was conducted within the framework of the project DODfast (Demonstration of Optical Data link fast), a cooperation of the German Aerospace Center (DLR) Institute of Communications and Navigation, Airbus Defence \& Space and ViaLight Communications. The goal of the demonstration is an optical downlink from the fast flying platform, a Panavia Tornado, to the ground station, DLR's Transportable Optical Ground Station (TOGS) during a flyby. This data dump demands short acquisition times of the data link and a high data-rate. The flight campaign was prepared and conducted together with the Bundeswehr Technical and Airworthiness Center (WTD 61) in Manching.

Unmanned/Unattended Sensors and Sensor Networks X, edited by Edward M. Carapezza,

Panos G. Datskos, Christos Tsamis, Proc. of SPIE Vol. 9248, 92480R · (c) 2014 SPIE

CCC code: $0277-786 X / 14 / \$ 18 \cdot$ doi: $10.1117 / 12.2067248$

Proc. of SPIE Vol. 9248 92480R-1 
The paper is structured as follows: chapter 1 gives the motivation and background of the experiment and briefly surveys the status quo of free-space optical aeronautical communications experiments. Chapter 2 outlines the basic system and experiment design. Chapter 3 contains description of execution, results and discussion of the flight campaign and chapter 4 draws the conclusions of the work.

A broad variety of research programs employed various flight platforms to test and demonstrate the capability of future optical aeronautical communications. General design rules of airborne optical terminals are discussed in [1]. We identify three different variations of airborne optical terminals to date: (1) turret integrated terminals, (2) cabin installed terminals and (3) fuselage integrated terminals. In the first case, usually a standard turret hosts the whole laser terminal and takes care of the coarse pointing and vibration compensation. The second case applies a more experimental setup where the laser beam propagates either through an open hole, door or an optical window. In the third case, the terminal is integrated in the aircraft body with minimum parts exposed to the air stream. Tracking tests with a cabin installed terminal transmitting through the window of a Boeing 767-200 are reported in [2], data-transmission from a Twin Otter aircraft in [3], [4] and validation tests of a space-terminal installed in a similar manner in a Cessna 206 aircraft are described in [5]. A modified Wescam turret integrated in the nose of a BAC 1-11 aircraft for a $200 \mathrm{~km}$ aircraft-mountain link is described in [6] and further turret designs are demonstrated with an Altair Unmanned-Aerial-Vehicle [7] and a Twin Otter aircraft [8]. Alternatively to the turret and cabin design, one can employ the fuselage integrated design (number 3) and thus install it inside the aircraft body as explained in [9] for an experimental system. Here, an optical glass dome protects the coarse pointing assembly (CPA) of this design and a Coudé path ducts the laser beam to a box inside the cabin of a Dornier Do 228. The airborne terminal (Micro Laser Terminal: MLT) used in our experiment is based on this solution. However, further development made it possible to miniaturize the glass dome, the CPA, the main terminal unit and enabled integration in the Avionic Demonstrator Tornado (ADT) Pod of a Tornado jetfighter. The distinction and goal of our demonstration to others is the targeted flight speed of 0.7 Mach, showing the capability of the fuselage integrated terminal design to cope with the appearing disturbances (shock and vibration) and use of a standalone transportable optical ground station as globally deployable receiver station. All three issues bring the technology to a maturity close to operational use.

\section{EXPERIMENT DESIGN}

Link budget analysis determined the feasibility of the experiment, under the given boundary conditions, and the design, in that way defining the possible flight heights, distances and specifications of the flight terminal MLT and the ground station TOGS. The most relevant system parameters are given in Table 1. The TOGS engages a primary mirror aperture of $600 \mathrm{~mm}$ with secondary mirror of $158 \mathrm{~mm}$ in a Ritchey-Chretien telescope design [10]. The different Field of Views (FoVs) of the tracking sensor and the Receiver-Font-End (RFE) are given by the telescope magnification, a subsequent common collimation lens and two individual focus lenses. The data-rate is $1.25 \mathrm{Gbit} / \mathrm{s}$ with intensity modulation, direct detection and on-off keying (IM/DD OOK). A free-space RFE with high sensitivity is chosen. To support optical tracking on MLT-side, two high power laser beacons operating in C-band are installed aligned to the TOGS telescope.

Table 1. TOGS, MLT and scenario parameters for the DODfast experiment used for the link budget analysis and experiment design.

\begin{tabular}{|c|c|c|}
\hline \multirow[t]{6}{*}{ TOGS } & Rx-aperture (diameter) & $600 \mathrm{~mm}$ \\
\hline & Rx-aperture obscuration (diameter) & $158 \mathrm{~mm}$ \\
\hline & FoV tracking sensor & $4.8 \mathrm{mrad}$ x $3.8 \mathrm{mrad}$ \\
\hline & FoV RFE & $166 \mu \mathrm{rad}$ \\
\hline & Sensitivity RFE@1.25 Gbit/s & $-35 \mathrm{dBm}$ \\
\hline & Wavelength uplink (beacon) & C-Band \\
\hline \multirow[t]{3}{*}{ MLT } & Full divergence angle $\left(1 / \mathrm{e}^{2}\right)$ & $2.26 \mathrm{mrad}$ \\
\hline & Maximum tolerable tracking error & $200 \mu \mathrm{rad}$ \\
\hline & Wavelength downlink (data) & C-Band \\
\hline \multirow[t]{4}{*}{ Scenario } & Data-rate (IM/DD OOK) & $1.25 \mathrm{Gbit} / \mathrm{s}$ \\
\hline & Observer height (TOGS) & $350 \mathrm{~m}$ \\
\hline & Atmospheric profile & Mid-latitude summer \\
\hline & $C_{\mathrm{n}}^{2}$-ground value for $\mathrm{HV}$-model & $1.7 \mathrm{e}-13 \mathrm{~m}^{-2 / 3}$ \\
\hline
\end{tabular}


The MLT also employs a transmitter in C-band. Beam divergence is selected to be large enough to maintain a stable link, to best cope with the aircraft vibrations, and low enough for minimal free-space loss. A conservative design approach led to a tolerable tracking error of $200 \mu \mathrm{rad}$ and beam divergence of $2.26 \mathrm{mrad}$ which fully serves the purpose of the demonstration. However, much margin for optimization in subsequent designs and implementations is given here on purpose. The observer height of the TOGS is $350 \mathrm{~m}$ MSL (location Manching, Germany). To support the conservative link budget, an atmospheric profile for summer time and daytime $C_{\mathrm{n}}^{2}$-ground value is used.

The link budget analysis revealed a link margin of $11.0 \mathrm{~dB}$ to $12.0 \mathrm{~dB}$ for flight height of $3 \mathrm{~km}$ and horizontal distance of $20 \mathrm{~km}$ and $-0.9 \mathrm{~dB}$ to $0.9 \mathrm{~dB}$ for $40 \mathrm{~km}$ distance which depends on the given visibility of $23 \mathrm{~km}$ or $50 \mathrm{~km}$, respectively. Therefore, the system is expected to perform until link distance of almost $40 \mathrm{~km}$. Static and stochastic losses are considered in the link budget. The first cover the deterministic attenuation of free-space loss, atmospheric extinction, optics loss and background light loss. The second covers signal fading due to scintillation and tracking errors and diode coupling loss due to focal spot speckles.

The DODfast system includes the Micro Laser Terminal, the Transportable Optical Ground Station and the sensor payload. The block diagram is given in Figure 1. The MLT is installed in the ADT-Pod attached to the Tornado. A remote ground station monitors and controls function and performance of the terminal. A mission radiation switch is installed in the cockpit for the sake of laser safety. The payload data signal is fed to the MLT with a standard single mode fiber (SMF). The TOGS receives the data signal and forwards it to the data sink. An up- and downlink in UHFand S-band, respectively, serve for TM/TC (Telemetry/Telecommand) communication to command the MLT and receive status and position information from the aircraft. The link acquisition is achieved on both sides based on knowledge of the counter terminals GPS position. The aircraft position is made available to the TOGS via the TM/TC link. Furthermore, optical tracking is performed on both sides to achieve and maintain precise beam pointing: the TOGS uses the data signal as beacon reference, whereas the MLT uses a dedicated low frequency modulated laser beacon.

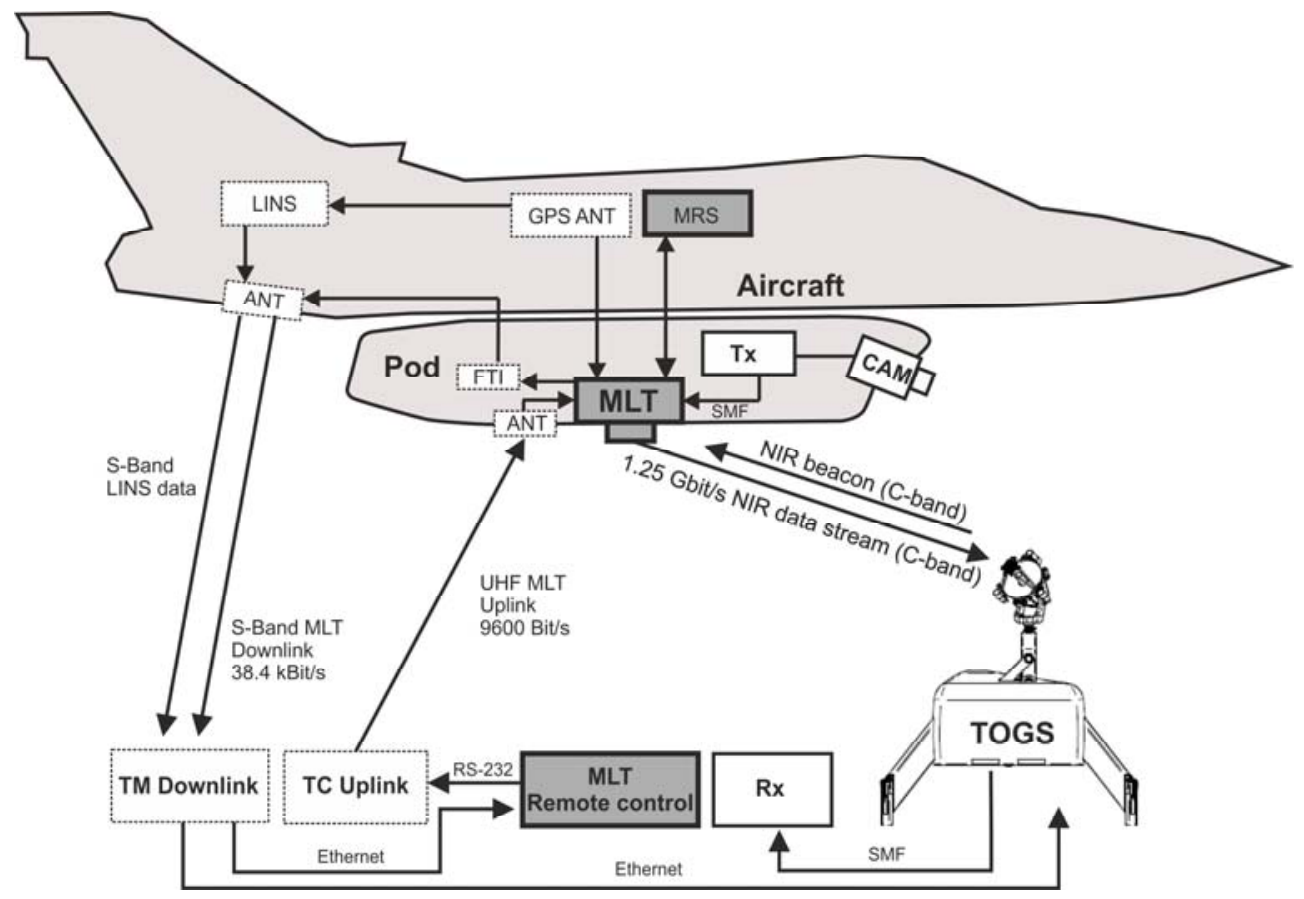

Figure 1. Overview of DODfast system. The aircraft and the attached pod are in bright gray with solid lines. The blocks of the payload camera, the data communication chain and the ground station are white with thin solid lines (CAM, Tx, TOGS, $\mathrm{Rx}$ ). The Micro Laser Terminal, its remote control station and the mission radiation switch are colored in dark gray with thick solid lines (MLT, MLT Remote control, MRS). The TM/TC and FTI (Flight Test Instrumentation) system is in white with thin dashed lines (GPS ANT, LINS, 2xANT, FTI, TM Downlink, TC Uplink). 


\section{FLIGHT CAMPAIGN}

\subsection{Execution}

The flight campaign took place near the Airbus Defense \& Space site in Manching, Germany in November 2013. The TOGS was located on the drop zone and aircraft take-off was at the Ingolstadt-Manching Airport, both neighboring the Airbus premises. Since lasers were applied on aircraft and ground side, laser safety regulations had to be taken care of by technical and organizational means. Figure 2 shows an image of the TOGS in front of the supply house that hosted place for the ground infrastructure and operations staff. The TM/TC uplink and downlink antenna were placed on the rooftop. Each flight lasted around two hours and was organized in separate runs, each having different flight parameters, i.e. flight path (circular, tangential, radial), height and speed. Pointing calibration of TOGS was performed on site by star referencing. The MLT got instantaneous heading information from an installed inertial measurement unit. Figure 3 depicts the Tornado in flight (left image). The white ADT-Pod is attached to the lower fuselage. The upper right image contains a zoom on the pod showing the small MLT glass dome. In the lower right, the glass dome is uninstalled for inspection of the CPA.

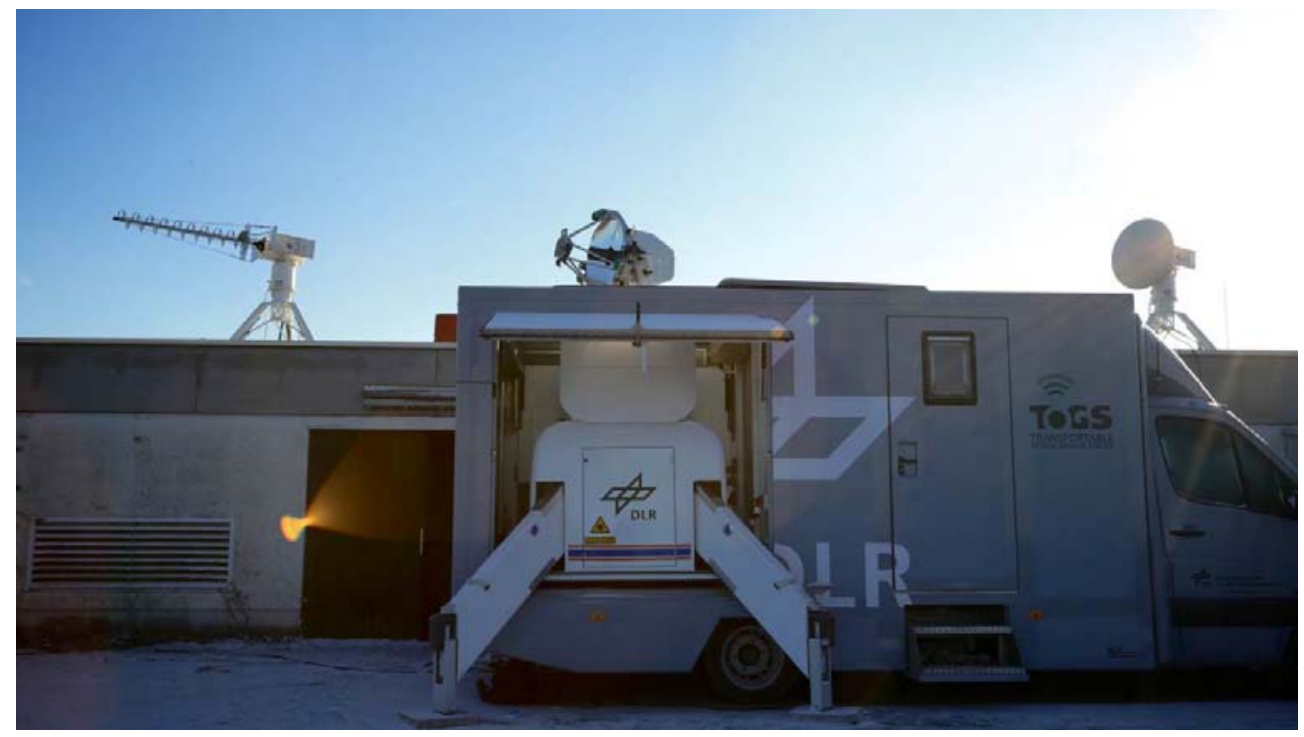

Figure 2. Image of the ground station setup at the test site in Manching. The TOGS telescope is unfolded and stands together with its van in front of the laser link ground command center. The TM/TC up- and downlink antennas are located on the rooftop of the building (Source: DLR).

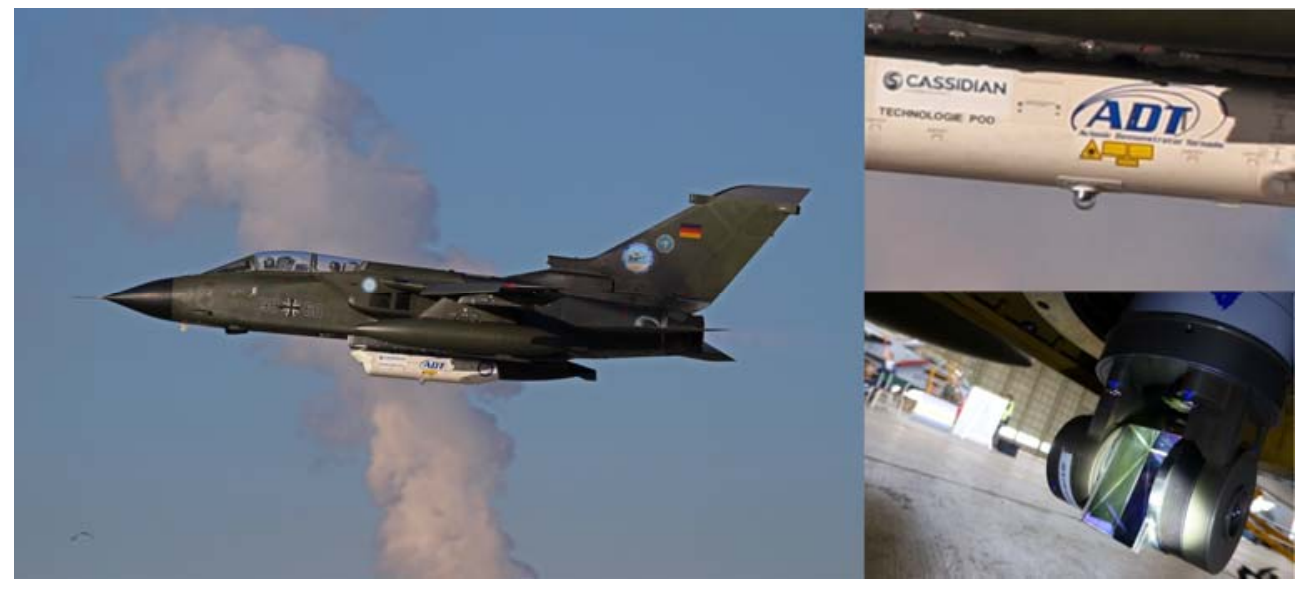

Figure 3. Left: Tornado with attached ADT-Pod during the second flight test. The white long box is the ADT-Pod with integrated MLT. (Source: Josef Gietl/Airbus Defense \& Space). Upper right: close-up of the ADT-Pod revealing the MLT dome. Lower right: MLT CPA without glass dome during inspection (Source: ViaLight Communications). 


\subsection{Results}

Tracking lock was achieved and video data was received during two flights. The weather condition during flight \#01 was very cloudy (overcast). However, during some low approach maneuvers, small cloud gaps could be used to establish a data link for a short time. Flight \#02 had better weather conditions (almost clear sky with hazy conditions and isolated clouds). Links could be established in all 13 runs of flight \#02 with aircraft speed of up to 0.7 Mach (maximum targeted speed), maximum tracking distance of $79 \mathrm{~km}$ and data transmission of over $50 \mathrm{~km}$. Therefore, the performance predicted by the conservative link budget $(<40 \mathrm{~km})$ was beaten by far. Table 2 lists the duration of active tracking for the MLT CTS (Coarse Tracking Sensor) and FTS (Fine Tracking Sensor) and the TOGS TS (Tracking Sensor) for flight \#02. Furthermore, duration of data signal lock is measured with an RSSI (Received Signal Strength Indication) signal from the RFE. The durations are listed for the individual runs and the total experiment.

Table 2. Overview of link times for the individual flight runs and the total experiment time during flight \#02. All 13 runs are listed with duration of the individual runs and duration of signal acquisition of the MLT CTS (Coarse Tracking Sensor), MLT FTS (Fine Tracking Sensor), TOGS TS (Tracking Sensor), and RFE (Receiver-Front-End).

\begin{tabular}{|c|c|c|c|c|c|}
\hline Run No. & Run duration & MLT CTS [s] & MLT FTS [s] & TOGS TS [s] & RFE [s] \\
\hline 01 & 313 & 290 & 290 & 275 & 148 \\
\hline 02 & 224 & 224 & 224 & 187 & 173 \\
\hline 03 & 261 & 175 & 170 & 137 & 91 \\
\hline 04 & 590 & 555 & 555 & 529 & 429 \\
\hline 05 & 245 & 65 & 65 & 65 & 38 \\
\hline 06 & 556 & 525 & 520 & 479 & 272 \\
\hline 07 & 619 & 554 & 435 & 374 & 329 \\
\hline 08 & 529 & 515 & 480 & 339 & 186 \\
\hline 09 & 692 & 630 & 605 & 580 & 238 \\
\hline 10 & 602 & 520 & 500 & 453 & 406 \\
\hline 11 & 151 & 135 & 135 & 127 & 109 \\
\hline 12 & 475 & 425 & 410 & 261 & 209 \\
\hline 13 & 450 & 430 & 385 & 414 & 349 \\
\hline Total & 5707 & 5043 & 4774 & 4220 & 2977 \\
\hline Fractional & & $88 \%$ & $84 \%$ & $74 \%$ & $52 \%$ \\
\hline
\end{tabular}

\subsection{Discussion}

The total experiment time of $5707 \mathrm{~s}$ is the sum of the run durations. The MLT CTS is the first sensor in the acquisition and tracking chain which receives a signal. Therefore, fractional link time is highest for this sensor (88 \%). The second sensor is the MLT FTS with nearly similar link times (84 \%). When MLT CTS and FTS have a valid signal, the MLT is in tracking mode and signal is expected on the TOGS sensors. In most cases, it took only seconds for the MLT to acquire the ground station signal. Deviation from this acquisition pattern can be only seen in run 7, 8, 9 and 13 . This is mostly because the signal strength for the MLT FTS was out of the expected range and not counted. The TOGS FT achieved $74 \%$ link time. Eventually, data signal lock was achieved $52 \%$ of experiment time which is defined by the RSSI exceeding a certain threshold. The reasons for not having $100 \%$ link time in all sensors are twofold: first, line-of-sight was blocked several times by a few cloud fields and by the aircraft body during high bank maneuvers. Second, a few technical issues are responsible: the fully operational automated power control on MLT side could not be implemented yet due to given certification processes. Therefore, the Tx-power was adjusted manually via TM/TC which sometimes could not be done just in time. Thus, the RFE occasionally was saturated leading to signal break down. Similar happened in the beacon uplink. Saturation of the MLT FTS during acquisition phase triggered the tracking algorithm to label a detected spot as invalid and therefore leading to acquisition delay. Next, signal delay in transmission of aircraft GPS position to TOGS resulted in a systematic blind pointing offset of the beacon laser. Delay compensation was implemented but needed to be optimized during runtime because the absolute time lag could not be determined beforehand. This additionally retarded the acquisition process. One occasion could be identified when MLT had a false spot detection for a short time period which caused delay of signal acquisition by TOGS. Also defocus on the TOGS sensors hindered perfect acquisition sometimes due to the need for manual readjustment of the focus. 


\section{CONCLUSION}

We successfully demonstrated a high-rate optical downlink from a fast flying platform, i.e. with a speed of 0.7 Mach. An integrated airborne optical terminal was used as transmitter for payload data, a transportable optical ground station as receiver station. Except for the laser safety regulations, operation of the flight terminal could be done without implications on the aircraft operation. The demonstration shows that integrated laser terminals (i.e. without additional stabilizing turret) are feasible and efficient to use for free-space optical communications even at vibration spectra typical for high jet fighter speeds. Furthermore, the use of compact transportable ground stations for this challenging application is also practical. The applied C-band DWDM technology for the IM/DD OOK and tracking systems has been proven to be suitable for the demonstrated scenario. The total fractional signal lock times of the tracking sensors of the MLT (88 \% and $84 \%$ ) and the TOGS (74\%) and the data receiver (52\%) shows that the applied technology is highly skilled for the addressed scenario. On the way to operational deployment, we identified several subsystems that affect acquisition performance the most like automatic power control on aircraft and ground station side, automated focus control and unknown/unsteady delay in aircraft position transmission. The upcoming challenges are now to refine and improve the actual implementations and system designs of the ground station and airborne terminal based on the knowledge gain from the experiment to cope up with the demands of operational systems and the link budget for higher data-rates. Next, transmission rates in the order of $40 \mathrm{Gbit} / \mathrm{s}$ and beyond are addressed, to fully exploit the capabilities of free-space optical aeronautical communications with DWDM technology.

\section{ACKNOWLEDGEMENTS}

The authors would like to thank all persons who were involved in the project DODfast and the conducted flight campaign. Special thanks goes to Mr. Marco Bobbio and DLR SHT, who contributed a lot to the project success. We also would like to thank all members from Airbus Defence \& Space who were involved in the TM/TC-link setup, MLT integration and the flight campaign. Finally we would like to give special thanks to the team of the Bundeswehr Technical and Airworthiness Center for Aircraft (WTD 61) in Manching who supplied the aircraft and gave tremendous support in all phases of the demonstration.

\section{REFERENCES}

[1] H. Henniger and D. Giggenbach, “AVIONIC OPTICAL LINKS FOR HIGH DATA-RATE COMMUNICATIONS," in Proceedings of 25th Congress of International Council of the Aeronautical Sciences, 2006.

[2] V. J. Chan and R. L. Arnold, "Results of one GBPS aircraft-to-ground lasercom validation demonstration,” in Proc. SPIE 2990, Free-Space Laser Communication Technologies IX, 1997, vol. 2990, pp. 52-59.

[3] F. G. Walther, S. Michael, R. R. Parenti, and J. A. Taylor, "Air-to-ground lasercom system demonstration design overview and results summary,” in Proc. of SPIE (Free-Space Laser Communications X), 2010, vol. 7814, p. 78140Y-78140Y-9.

[4] F. G. Walther, G. A. Nowak, S. Michael, R. Parenti, J. Roth, J. Taylor, W. Wilcox, R. Murphy, J. Greco, J. Peters, T. Williams, S. Henion, R. Magliocco, T. Miller, and A. Volpicelli, "Air-to-ground lasercom system demonstration," in Proceedings of the Military Communications Conference, 2010, pp. 2295-2301.

[5] H. Hemmati and J. M. Kovalik, "10 Gb/s Lasercom Terminal for Satellites," in Proc. of International Conference on Space Optical Systems 2012, 2012.

[6] L. B. Stotts, "Optical communications in atmospheric turbulence," in Proc. of SPIE, Free-Space Laser Communications IX, 2009, vol. 7464.

[7] G. G. Ortiz, S. Lee, S. P. Monacos, M. W. Wright, and A. Biswas, "Design and development of a robust ATP subsystem for the Altair UAV-to-ground lasercomm 2.5-Gbps demonstration," in Proc. SPIE 4975 (Free-Space Laser Communication Technologies XV), 2003, vol. 4975.

[8] L. C. Andrews, R. L. Phillips, R. Crabbs, T. Leclerc, and P. Sauer, "Final report: channel characterization for free-space optical communications,” UCF DARPA, 2012.

[9] J. Horwath and C. Fuchs, "Aircraft to ground unidirectional laser-communications terminal for high-resolution sensors,” in Proc. SPIE, Free-Space Laser Communication Technologies XXI, 2009, vol. 7199.

[10] C. Fuchs, M. Brechtelsbauer, J. Horwath, A. Shrestha, F. Moll, D. Giggenbach, and C. Schmidt, "DLR's Transportable Optical Ground Station," in Proc. of Applications of Lasers for Sensing and Free Space Communications (OSA Technical Digest ), 2013. 